Pacific Journal of Mathematics

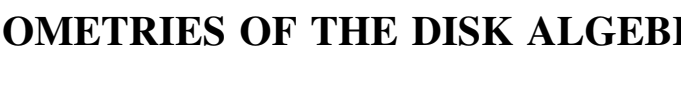




\section{ISOMETRIES OF THE DISK ALGEBRA}

\section{J. N. MCDonald}

In this paper we are concerned with the problem, posed by R. R. Phelps, of describing the into isometries of the disk algebra. We show that, in a certain sense, every isometry can be approximated by convex combinations of isometries of the form $f \rightarrow k(f \circ \phi)$. We also give some sufficient conditions for an isometry to be of the form $f \rightarrow k(f \circ \phi)$.

Let $D$ and $\Gamma$ denote, respectively, the open unit disk and the unit circle. The disk algebra, i.e., the algebra of all complex valued functions which are continuous on $D \cup \Gamma$ and analytic on $D$, will be denoted by $A$. It will be assumed that $A$ is equipped with the sup-norm.

Operators of the form

$$
T f=k(f \circ \phi)
$$

are isometries of $A$ : if $k \in A$, if $\|k\|=1$, and if $\phi: D \cup \Gamma \rightarrow D \cup \Gamma$ is analytic on $D$, continuous on $D \cup \Gamma \sim k^{-1}(0)$, and satisfies $\phi\left(k^{-1}(\Gamma)\right) \supset \Gamma$. In fact, if $T$ is a surjective linear isometry of $A$, then it must be of the form (1) with $k$ being a constant, and $\phi$ being a Mobius transformation. (See [3, pp. 142-148].) Rochberg [8] has shown that if $T$ is an isometry such that $T 1=1$, and $T(A)$ is a sub-algebra of $A$, then $T$ is of the form (1) with $k \equiv 1$.

Note that any bounded linear operator $T: A \rightarrow A$ which satisfies (1) also satisfies.

$$
T 1 T(f g)=T f T g
$$

for all $f$ and $g$ in $A$. Moreover, we have the following.

Proposition 1.1. A bounded linear operator $T: A \rightarrow A$ satisfies (2) for all $f, g \in A$ iff it is of the form (1).

Proof. It is only necessary to show that, if $T$ satisfies (2) for all $f, g \in A$, then it satisfies (1).

Suppose that $w$ is a point of $D$ where $T 1$ is not 0 . Consider the linear functional defined on $A$ by

$$
L_{w}(f)=(T 1(w))^{-1} T f(w) .
$$

By (2), $L_{w}$ is a multiplicative. Hence, there is a $v$ in $D \cup \Gamma$ such that 
$L_{w}(f)=f(v)$. Since $v=(T 1(w))^{-1} T Z(w)$, where $Z$ is the identity function on $D \cup \Gamma$, it follows that the function $\phi=(T 1)^{-1} T Z$ is bounded on $D$. Thus, the singularities of $\phi$ in $D$ are removable. Let $S$ be the operator defined on $A$ by

$$
S f=T 1(f \circ \phi) .
$$

It follows easily from (2) that $S Z^{n}=T Z^{n}$ for $n=0,1, \cdots$. Since the polynomials in $Z$ are dense in $A$, the operators $T$ and $S$ are the same. If $T 1 \equiv 0$, then, by (2), $(T f)^{2}=T 1 T f^{2} \equiv 0$. It follows that $T$ is of the form (1) with $k \equiv 0$.

For an example of an isometry which fixes 1 but is not multiplicative, see [8].

For the remainder of this section, $T$ will denote an arbitrary isometry of $A$. Consider the closed set $\Gamma(T)=\{z \in \Gamma|| T l(z) \mid=1$ and there is a point $\hat{T}(z)$ in $\Gamma$ such that $T f(z)=T 1(z) f(\hat{T}(z))$ for all $f \in A\}$. Since $A$ separates the points of $\Gamma$, it follows that the mapping $z \rightarrow \hat{T}(z)$, denoted by $\hat{T}$, is well defined and continuous on $\Gamma(T)$. In [5], we showed that $\hat{T}$ maps $\Gamma(T)$ onto $\Gamma$. The following proposition gives a simple description of $\Gamma(T)$.

PROPOSITION 1.2.

$$
\Gamma(T)=\{w \in \Gamma|| T 1(w) \mid=1 \quad \text { and } \quad|T Z(w)|=1\} .
$$

Proof. It is enough to show that if $\left|T 1\left(z_{1}\right)\right|=\left|T Z\left(z_{1}\right)\right|=1$, then $z_{1} \in \Gamma(T)$. By the Hahn-Banach theorem, there is a measure $\mu$ on $\Gamma$ having total variation $\leqq 1$ such that $\operatorname{Tf}\left(z_{1}\right)=\int f d \mu$ for all $f \in A$. Let $a=\int 1 d \mu$ and $b=\int Z d \mu$, where $Z$ is the identity on $D \cup \Gamma$. Since $\bar{a} \mu$ has total variation $\leqq 1$ and $\int \bar{a} d \mu=1$, it follows that $\bar{a} d \mu$ is nonnegative. Note that $\int \operatorname{Re}(1-a \bar{b} Z) \bar{a} d \mu=0$. Thus, $\operatorname{Re}(1-a \bar{b} Z)$ is 0 on the support of $\mu$. Hence the support of $\mu$ consists of a single point, i.e., $\hat{T}\left(z_{1}\right)$.

THEOREM 1.1. Suppose $m(\Gamma(T))>0$, where $m$ denotes Lesbegue measure on $\Gamma$. Then $T$ is of the form (1).

Proof. For $f, g \in A$, we have

$$
T 1(z) T(f g)(z)=T f(z) T g(z)
$$


for every $z \in \Gamma(T)$. Any two functions in $A$ which agree on a subset of $\Gamma$ having positive Lesbeque measure are equal. (See $[3$, p. 52].) Thus $T 1 T(f g)=T f T g$. It follows by Proposition 1.1 that $T$ is of the form (1).

THEOREM 1.2. Assume that $T 1$ is an inner function. Suppose that $T(A)$ contains a function $G$ having the following properties: $\|G\|=1$, $\left.m\left(G^{-1}\right)(\Gamma)\right)>0$, the set of connected components of $G^{-1}(\Gamma)$ is countab $e$, and $G$ is not a constant multiple of $T 1$. Then $T$ is of the form (1).

Proof. Let $H=\overline{T 1} G$. Note that $H^{-1}(\Gamma)=G^{-1}(\Gamma)$. Let $\left\{J_{1}, J_{2}, \cdots\right\}$ denote the collection of connected components of $H^{-1}(\Gamma)$. Suppose it can be shown that, for some $q, m\left(H\left(J_{q} \cap \Gamma(T)\right)\right)>$ 0 . Then $J_{q}$ is necessarily a nontrivial sub-arc of $\Gamma$. By a form of the Schwartz reflection principle (See, e.g. [2, p. 187].), $G$ can be continued analytically across the interior of $J_{q}$. It follows that the restriction of $H$ to the interior of $J_{q}$ is continuously differentiable. If $H$ were constant on $J_{q}$, then we would have $G=c T 1$ where $c$ is a constant. Thus, $H$ is not constant and, hence, $m\left(J_{q} \cap \Gamma(T)\right)>0$. It now follows by Theorem 1.1 that $T$ is of the form (1).

It remains to be shown that $m\left(H\left(J_{q} \cap \Gamma(T)\right)\right)>0$ for some $q$. It is claimed that

$$
H\left(H^{-1}(\Gamma)\right)=H\left(H^{-1}(\Gamma) \cap \Gamma(T)\right) .
$$

For each $z \in \Gamma$, there exists a measure $\mu_{z}$, having total variation $\leqq 1$, such that $\int f d \mu_{z}=T f(z)$ for each $f \in A$. In particular, we have $1=$ $\overline{T 1(z)} d \mu_{z}$. It follows that the measure $\overline{T 1(z) \mu_{z}}$ is nonnegative. Suppose that $z$ is choosen so that $|G(z)|=|H(z)|=1$. Let $F$ be the unique function in $A$ such that $G=T F$. Then

$$
\int \operatorname{Re}(1-\overline{H(z)} F) \overline{T 1(z)} d \mu=0 .
$$

It follows that $H(z)=F(w)$ for each $w$ in the support of $\mu_{z}$. Since the mapping $\hat{T}$ is onto, there exists a $z_{1} \in \Gamma(T)$ such that

$$
\begin{aligned}
H(z) & =F\left(\hat{T}\left(z_{1}\right)\right) \\
& =\overline{T 1\left(z_{1}\right)} T 1\left(z_{1}\right) F\left(\hat{T}\left(z_{1}\right)\right) \\
& =H\left(z_{1}\right) .
\end{aligned}
$$


Next it is claimed that $m\left(H\left(H^{-1}(\Gamma)\right)\right)>0$. If $m\left(H\left(H^{-1}(\Gamma)\right)\right)=0$, then $H$ is constant on all of the $J_{n}$ 's. Since at least one of the $J_{n}$ 's is a nontrivial sub-arc of $\Gamma$, it follows that $G=c T 1$ for some constant $c-a$ contradiction to the hypothesis that $G$ not be a scalar multiple of $T 1$. Finally, we have

$$
0<m\left(H\left(H^{-1}(\Gamma)\right)\right) \leqq \Sigma m\left(H\left(J_{n} \cap \Gamma(T)\right)\right) .
$$

It follows that $m\left(H\left(J_{q} \cap \Gamma(T)\right)\right)>0$ for some $q$.

CoRollary. Suppose that $T 1$ is an inner function. If TA contains an inner function which is not a scalar multiple of $T l$ then $T$ is of the form (1).

Remark. Let $\mathscr{A}$ denote the sub-algebra of $A$ consisting of functions which are analytic in a neighborhood of $D \cup \Gamma$. By arguments similar to those used to prove Theorem 1.2, one can show that every isometry of $\mathscr{A}$ must be of the form (1).

2. Approximation of arbitrary isometries. As in the previous section, $T$ will denote an arbitrary isometry of $A$. Let $B$ denote the space of bounded linear operators: $A \rightarrow A$ and let $B_{1}$ denote the set of members of $B$ having norm $\leqq 1$. As in [5], we define $E(T)=\left\{U \in B_{1} \mid U f(z)=T f(z)\right.$ for every $z \in \Gamma(T)$ and every $f \in$ $A\}$. In [5] we showed that $E(T)$ is a face of $B_{1}$, that $E(T)$ is closed in the weak operator topology, and that each member of $E(T)$ is an isometry. Thus, the set of isometries of $A$ is the union of weak operator-closed faces of $B_{1}$. It follows from Proposition 1.2, that

$$
E(T)=\left\{U \in B_{1}|U Z| \Gamma(T)=T Z \mid \Gamma(T) \quad \text { and } \quad U 1|\Gamma(T)=T 1| \Gamma(T)\right\},
$$

where $Z$ denotes the identity function on $D \cup \Gamma$. If $m(\Gamma(T))>0$, it follows that $E(T)=\{T\}$. Suppose that $m(\Gamma(T))=0$. Let $A_{1}$ denote the unit ball in $A$, let $S_{1}=\left\{f \in A_{1}|f| \Gamma(T)=\hat{T}\right\}$, and let $S_{2}=$ $\left\{g \in A_{1}|g| \Gamma(T)=T 1 \mid \Gamma(T)\right\}$. By a result due to Rudin [9], both $S_{1}$ and $S_{2}$ have infinitely many members. Let $h \in S_{1}$ and $k \in S_{2}$. The operator $U$ defined by $U f=k(f \circ h)$ is in $E(T)$. Thus, $E(T)$ contains infinitely many elements iff $m(\Gamma(T))=0$. For the remainder of the paper, we will consider only isometries $T$ for which $m(\Gamma(T))=0$.

Let $F(T)=\{U \in E(T) \mid U$ is of the form (1)\}. In view of [5, Th. 3], it is natural to ask whether $E(T)$ is the closed convex hull of $F(T)$, where the closure is taken in the weak operator topology? Although we are unable to answer this question, we will show that there is a family $\subseteq$ 
of locally convex Hausdorff topologies on $B$ with the following properties: for each $\mathscr{T} \in \mathcal{S}, E(T)$ is the $\mathscr{T}$-closed convex hull of $F(T)$, and the weakest topology containing all the members of $\subseteq$ is the weak operator topology.

The weak operator topology on $B$ is the weakest topology in which all linear functionals of the form $H \rightarrow \int H f d \mu$, where $f$ is in $A$ and $\mu$ is a Baire measure on $\Gamma$, are continuous. It follows that the space $B^{*}$ of weak operator continuous linear functionals on $B$ is the direct sum of sub-spaces $\mathscr{A}$ and $\mathscr{Y}$, where $\mathscr{A}$ is the sub-space of $B^{*}$ spanned by linear functionals of the form $H \rightarrow \int(H f) g d m$ with $g \in L_{1}(m)$, and where $\mathscr{S}$ is the sub-space of $B^{*}$ spanned by functionals of the form $H \rightarrow \int H f d \nu$ with $\nu$ being singular with respect to $m$. (See [1, p. 421]). Let $L_{1}^{+}(m)$ denote $\left\{g \in L_{1}(m) \mid g \geqq 0\right.$ a.e. $\}$. For each $g \in L_{1}^{+}(m)$ we define the Sg-topology on $B$ to be the weakest topology in which the linear functionals of the form $H \rightarrow \int(H f) g d m$ with $f$ in $A$, and the linear functionals in $\mathscr{S}$, are continuous. Set $\subseteq=\left\{\mathscr{S g} \mid g \in L_{1}^{+}(m)\right\}$. Let $\mathscr{W}$ denote the weak operator topology on $B$. Note that $\mathscr{S g} \subseteq \mathscr{W}$ for each $g \in L_{1}^{+}(m)$. By [1, p. 421], the $\mathscr{S}$-continuous linear functionals on $B$ are those of the form $l(H)=\int H f g d m+\sum_{i=1}^{n} \int H f_{i} d \mu_{i}$, where the measures $\mu_{i}, i=1,2, \cdots, n$, are singular with respect to $m$ and $f, f_{1}, f_{2} \cdots f_{n} \in$ $A$. Let $U$ denote the smallest locally convex topology on $B$ which contains all members of $\mathfrak{S}$. Any functional of the form $L(H)=$ $\int H f d \nu$, where $f \in A$, and $\nu$ is a regular Baire measure, can be written in the form

$$
L(H)=\int H f d \mu+\sum_{n=1}^{4} \int H\left(i^{n} f\right) g_{n} d m
$$

where $\mu$ is singular with respect to $m$, and $g_{1}, g_{2}, g_{3}, g_{4} \in L_{1}^{+}(m)$. It follows that $L$ is $U$-continuous. Hence, by the definition of $\mathscr{W}$, we have $U \subseteq \mathcal{W}$.

THEOREM 2.1. For each $g \in L_{1}^{+}(m), E(T)$ is the $\mathscr{S}$-closed convex hull of $F(T)$.

REMARK. It is not possible to prove Theorem 2.1 by using arguments based on the Krein-Milman theorem. For in order for the Krein-Milman theorem to apply to $E(T)$ it would be necessary for 
$E(T)$ to be compact in the $\mathscr{S g}$-topology, but the following argument shows that $E(T)$ is not $\mathscr{S}$-compact for any $g \in L_{1}^{+}(m)$ : Let $K$ be a "Cantor" subset of $\Gamma$ which is disjoint from $\Gamma(T)$. Let $C_{1}(K)$ denote the set of continuous complex valued functions on $K$ having absolute value $\leqq 1$. Define $j: E(T) \rightarrow C_{1}(K)$ by $j(U)=U Z \mid K$. If $C_{1}(K)$ is equipped with the topology of pointwise convergence, then $j$ is $\mathscr{P g}$ continuous for each $g \in L_{1}^{+}(m)$. By [9], the map $j$ is onto. Since $C_{1}(K)$ is not compact in the topology of pointwise convergence, it follows that $E(T)$ is not compact in the $\mathscr{S g}$-topology.

Our proof of Theorem 3 will depend on the following two lemmas:

Lemma 2.1. For $z$ in $D$, and $t \in[-\pi, \pi]$ let

$$
P_{z}\left(e^{i t}\right)=\operatorname{Re}\left(\left(z+e^{i t}\right)\left(z-e^{i t}\right)^{-1}\right),
$$

i.e., $P_{z}\left(e^{t t}\right)$ is the Poisson kernel. Consider the set $V=\left\{\sum_{i=1}^{n} c_{i} P_{z_{i}} \mid c_{i} \geqq 0\right.$ and $z_{i} \in D$ for $\left.i=1,2, \cdots, n\right\}$. Then the $L_{1}$-closure of $V$ is $L_{1}^{+}(m)$.

Proof. Suppose that $g_{1} \in L_{1}^{+}(m)$, but $g_{1}$ is not in the closure of $V$. Then there exists an $h$ in $L_{\infty}(m)$, such that $\int g_{1} h d m>0$ and $\int v h d m \leqq 0$ for every $v \in V$. In particular

$$
\int P_{z}\left(e^{i t}\right) h\left(e^{i t}\right) d m\left(e^{i t}\right) \leqq 0
$$

for all $z$ in $D$. Fatou's Theorem [3, p. 34] implies that $h \leqq 0$ almost everywhere with respect to $m$. Hence, $\int g h d m \leqq 0$. Thus, we have reached a contradiction.

Lemma 2.2. Let $E$ be a closed subset of $\Gamma$ such that $m(E)=$ 0 . Let $\phi_{0}: E \rightarrow D \cup \Gamma$ be continuous. Consider $z_{1}, z_{2}, \cdots, z_{n}, w \in$ $D$. There is a function $\phi$ in the unit ball $A_{1}$ of $A$ which extends $\phi_{0}$ and satisfies $\phi\left(z_{i}\right)=w$ for $i=1,2, \cdots, n$.

Proof. Suppose that $w=0$. Let

$$
B(Z)=\prod_{\imath=1}^{n}\left[\left(z-\bar{z}_{\imath}\right)\left(1-\bar{z}_{i} z\right)^{-1}\right]
$$

For each $u$ in $\Gamma$, we have $|B(u)|=1$. Define $\beta_{0}$ on $E$ by $\beta_{0}(u)=$ $\overline{B(u)} \phi_{0}(u)$. The function $\beta_{0}$ has an extension $\beta$ in $A_{1}$. It follows that 
$B \beta$ satisfies the assertion of the lemma in the case where $w=0$. If $w \neq 0$, we choose a Mobius transformation $\tau$ of $D$ such that $\tau(0)=w$, and apply the preceeding argument to obtain a function $\phi_{1}$ in $A_{1}$ which extends $\tau^{-1} \circ \phi_{0}$ and maps $z_{1}, \cdots, z_{n}$ into 0 . Thus, the function $\phi=$ $\tau \circ \phi_{1}$, extends $\phi_{0}$, lies in $A_{1}$, and statisfies $\phi\left(z_{i}\right)=w$ for $i=1,2, \cdots n$.

Proof of Theorem 2.1. (The argument used here is an adaptation of one due to Morris and Phelps [6, Th. 2.1].)

Suppose $U \in E(T)$ but it is not in the $\mathscr{S}$-closed convex hull of $F(T)$. By [1, Th. 9, p. 421], there are functions $f, f_{1}, f_{2}, \cdots, f_{n} \in A$, measures $\mu_{1}, \mu_{2}, \cdots, \mu_{n}$ on $\Gamma$ which are singular with respect to $m$, and a real number $r>0$ such that

$$
\operatorname{Re}\left(\int(U f) g d m+\sum_{i=1}^{n} \int U f_{i} d \mu_{i}\right) \geqq \operatorname{Re}\left(\int(F f) g d m+\sum_{i=1}^{n} \int F f_{i} d \mu_{i}\right)+r,
$$

for every $F$ in $E(T)$.

By Lemma 2.1, there are points $z_{1}, z_{2}, \cdots, z_{p} \in D$ and nonnegative real numbers $c_{1}, c_{2}, \cdots, c_{p}$ such that

$$
\begin{gathered}
\operatorname{Re}\left(\sum_{j=1}^{p} c_{j} U f\left(z_{j}\right)+\sum_{i=1}^{n} \int U f_{i} d \mu_{i}\right) \\
\geq \operatorname{Re}\left(\sum_{j=1}^{p} c_{j} F f\left(z_{j}\right)+\sum_{i=1}^{n} \int F f_{i} d \mu \hat{i}\right)+\frac{r}{2},
\end{gathered}
$$

for every $F$ in $F(T)$. We can assume without loss of generality that $\mu_{i} \geqq 0$ for $i=1,2,, \cdots, n$. Since $U f_{i}=F f_{i}$ on $\Gamma(T)$ for $i=1,2, \cdots, n$ and $F \in F(T)$, we can also assume that $\mu_{i}(\Gamma(T))=0$ for $i=$ $1,2, \cdots, n$. Let $\nu=\sum_{i=1}^{n} \mu_{i}$. Given $\epsilon>0$, there is a closed subset $Y$ of $\Gamma \sim \Gamma(T)$ such that $m(Y)=0$ and $\nu(\Gamma \sim Y)<\epsilon$. Let $h_{i}$ denote the Radon-Nikodym derivative of $\mu_{i}$ with respect to $\nu$ for $i=$ $1,2, \cdots, n$. Choose continuous functions $h_{i}^{\prime}$ on $\Gamma$ such that $0 \leqq h_{i}^{\prime} \leqq 1$ and $\int\left|h_{i}-h_{i}^{\prime}\right| d \nu<\epsilon$ for $i=1,2, \cdots, n$.

Let $g=\sum_{i=1}^{n} h_{i}^{\prime} U f_{i \cdot} \quad$ For each $y \in \Gamma$, define $k_{y}=\sum_{i=1}^{n} h_{i}^{\prime}(y) f_{i}$. Then $g(y)=U k_{y}(y) . \quad g(y)$ is also equal to $\left(U^{*} e_{y}\right)\left(k_{y}\right)$, where $U^{*}$ is the adjoint of $U$ and $e_{y}$ represents the "evaluation at $y$ " functional on $A$. Let $S$ denote the unit ball in the dual space of $A$. Since $U^{*}$ maps $S$ into $S$, it follows that $U^{*} e_{y} \in S$. The function $W(\rho)=\operatorname{Re} \rho\left(k_{y}\right)$ is weak* continuous on $S$ and $\sup W(S) \geqq \operatorname{Re} U^{*} e_{y}\left(k_{y}\right)=\operatorname{Re} g(y)$. The extreme points of $S$ are exactly the functionals $c e_{y}$, where $c, y \in \Gamma$. It 
follows from the Krein-Milman theorem that, for each $y \in \Gamma$, there exist $\phi(y), c(y) \in \Gamma$, such that

$$
\operatorname{Re}\left(\sum_{i=1}^{n} c(y) h_{i}^{\prime}(y) f_{i}(\phi(y))\right)>\operatorname{Re} g(y)-\epsilon .
$$

For each $y \in Y$ choose an open neighborhood $V_{y}$ of $y$ such that $V_{y} \cap \Gamma(T)=\phi$ and

$$
\operatorname{Re}\left(\sum_{i=1}^{n} c(y) h_{i}^{\prime}(w) f_{i}(\phi(y))\right)>\operatorname{Re} g(w)-2 \epsilon
$$

for every $w \in V_{y}$. Let $\left\{V_{y_{1}}, \cdots, V_{y_{p}}\right\}$ be a finite collection of $V_{y}$ 's which covers $y$. We can easily find another open cover $\left\{U_{1}, \cdots, U_{p}\right\}$ of $Y$ such that $U_{i} \subset V_{y_{i}}$ and $\nu\left(\left\{y \mid y\right.\right.$ is in more than one $\left.\left.U_{j}\right\}\right)<\epsilon$. Consider the sets

$$
H_{j}=\left(Y \cap U_{j}\right) \sim \cup\left\{U_{i} \mid i \neq j\right\}, \quad j=1,2, \cdots, p .
$$

Then $H_{i}$ 's are closed and disjoint and $\nu\left(Y \sim \cup_{j=1}^{P} H_{j}\right)<\epsilon$.

Define mappings $\theta_{0}, k_{0}: \Gamma(T) \cup\left[\cup_{i=1}^{P} H_{i}\right] \rightarrow \Gamma$ by

$$
\begin{gathered}
\theta_{0}(y)=\left\{\begin{array}{lll}
\phi\left(y_{j}\right) & \text { if } & y \in H_{j} \\
\hat{T}(y) & \text { if } & y \in \Gamma(T) .
\end{array}\right. \\
k_{0}(y)=\left\{\begin{array}{lll}
c\left(u_{j}\right) & \text { if } & y \in H_{j} \\
T 1(y) & \text { if } & y \in \Gamma(T) .
\end{array}\right.
\end{gathered}
$$

Note that $m\left(\Gamma(T) \cup\left[\cup_{i=1}^{P} H_{j}\right]\right)=0$. Since $U$ is an isometry, there are points $w_{0}$ and $w_{1}$ in $D$ such that $\operatorname{Re} w_{0} f\left(w_{1}\right) \geqq \operatorname{Re} U f\left(z_{i}\right)-\epsilon$ for $i=$ $1,2, \cdots, p$. By Lemma 2.2, there are extensions $\theta$ and $k$, of $\theta_{0}$ and $k_{0}$ respectively, which lie in $A_{1}$ and satisfy $\theta\left(z_{j}\right)=w_{1}$ and $k\left(z_{j}\right)=w_{0}$ for $j=1,2, \cdots, n$. Define the linear operator $F_{1}: A \rightarrow A$ by $F_{1} h=$ $k(h \circ \theta)$. Clearly, we have $F_{1} \in F(T)$. By a straightforward argument, we can find a constant $M>0$ independent of $\epsilon$ such that

$$
\begin{gathered}
\operatorname{Re}\left(\sum_{j=1}^{p} c_{j} F_{1} f\left(z_{j}\right)+\sum_{i=1}^{n} \int F_{1} f_{i} d \mu_{i}\right) \\
>\operatorname{Re}\left(\sum_{j=1}^{p} c_{j} U f\left(z_{j}\right)+\sum_{i=1}^{n} \int U f_{i} d \mu_{i}\right)-M \epsilon .
\end{gathered}
$$

We can obtain a contradiction to (3) by taking $\epsilon$ to be sufficient small. 
Corollary 2.1. Suppose $T 1 \equiv 1$. Let $E_{1}(T)=\{U \mid U \in E(T)$ and $U 1=1\}$, and let $F_{1}(T)=E_{1}(T) \cap F(T)$. Then for each $g \in L_{1}^{+}(m)$, the set $E_{1}(T)$ is the closed convex hull of $F_{1}(T)$ where the closure is taken in the $\mathscr{Y g}$-topology.

Proof. Let $S_{1}=\{L \in S \mid L(1)=1\}$. The adjoint $T^{*}$ of $T$ maps $S_{1}$ to $S_{1}$. The extreme points of $S_{1}$ are the functionals of the form $e_{y}$ with $y \in \Gamma$. Thus, in the proof of Theorem 2.1 we may take $c(y)=$ 1. Also, it is clear that, in this case, we may take $w_{0}=$ 1. Consequently, it can be asumed that the function $k$ is identically 1 .

3. The case $T 1=1$. In this section it will be assumed that $T 1=1$. We will investigate the closure in the weak operator topology of the set $\operatorname{cov} F_{1}(T)$.

Let $H_{\infty}$ denote the space of bounded analytic functions on $D$ and let $B\left(H_{\infty}\right)$ denote the space of bounded linear operators on $H_{\infty}$. Denote by $\mathscr{P}$ the weakest topology on $B\left(H_{\infty}\right)$ such that all linear functionals of the form $M \rightarrow M g(z)$, where $g \in H_{\infty}$ and $z \in D$, are continuous. The following property of $B\left(H_{\infty}\right)$ will be very useful in this section: The unit ball of $B\left(H_{\infty}\right)$ is $\mathscr{P}$-compact. To verify this property it sufficies to use a result due to Kadison [4] together with the fact that the unit ball $H_{\infty}^{1}$ of $H_{\infty}$ is compact in the topology of pointwise convergence.

Let $A_{T}=\left\{\phi \in A_{1}|\phi| \Gamma(T)=\hat{T}\right\}$. Let $H_{T}$ denote the closure of $A_{T}$ in the topology of pointwise convergence on $D$. Since $H_{T} \subseteq H_{\infty}^{1}$, it follows that $H_{T}$ is compact in the topology of pointwise convergence on $D$. Each $F \in F_{1}(T)$ is of the form $F f=f \circ \phi$ for all $f \in A$, where $\phi \in A_{T}$. Thus, $F$ has an extension to $H_{\infty}$ denoted $F^{*}$ which is defined by $F^{*} g=g \circ \phi$ for every $g \in H_{\infty}$. Similarly, each $V \in \operatorname{cov} F_{1}(T)$ has an extension $V^{*}$ lying in $\operatorname{cov} F_{1}^{*}(T)$, where $F_{1}^{*}(T)=$ $\left\{F^{*} \mid F \in F_{1}(T)\right\}$. Since $F_{1}^{*}(T)$ is contained in the unit ball of $B\left(H_{\infty}\right)$, it

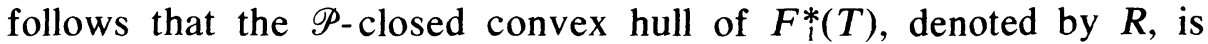
compact in the $\mathscr{P}$-topology. Let $Q$ denote the $\mathscr{P}$-closure of $F_{1}^{*}(T)$. Suppose that $W \in R$. By the integral form of the KreinMilman Theorem [7, p. 6], there is a probability measure $\mu_{W}$ supported by $Q$ such that

$$
W g(z)=\int_{Q} W^{\prime} g(z) d \mu_{W}\left(W^{\prime}\right)
$$

for every $g \in H_{\infty}$ and every $z \in D$. Note that $Q=\{W \mid W g=g \circ \phi$, where $\left.\phi \in H_{T}\right\}$. Thus, $Q$ may be identified with $H_{T}$. Consequently, we can write

$$
W g(z)=\int_{H_{T}} g \circ \phi(z) d \mu_{W}(\phi)
$$


for all $g \in H_{\infty}$ and all $z \in D$. Suppose now that $U$ is in the weak operator closed convex hull of $F_{1}(T)$. Then there exists a net $\left\{V_{\alpha}\right\}$ in cov $F_{1}(T)$ which converges in the weak operator topology to $U$. In particular, $U f(z)=\lim V_{\alpha} f(z)$ for each $f \in A$ and each $z \in D$. The net $V_{\alpha}^{*}$ has a subnet $V_{\beta}^{*}$ which converges to some $U^{*} \in R$. By the definition of the $\mathscr{P}$-topology, we have $U^{*} f(z)=U f(z)$ for $f \in A$ and $z \in D$. Thus, we have proved the following:

THEOREM 3.1. Let $U$ be in the closure of $\operatorname{cov} F_{1}(T)$ in the weak operator topology. Then there exists a probability measure $\mu$ on $H_{T}$ such that

$$
U f(z)=\int_{H_{T}} f \circ \phi(z) d \mu(\phi)
$$

for each $f \in A$ and each $z \in D$.

We will now use Theorem 3.1 to derive another sufficient condition for an isometry to be of the form (1).

THEOREM 3.2. Suppose $U$ is in the weak operator closure of $\operatorname{cov} F_{1}(T)$. If there is a nonconstant inner function $G$ such that $U G$ is an extreme point of $A_{1}$, then $U$ is of the form (1).

Our proof of Theorem 3.2 depends upon the following technical lemma.

LEMMA 3.1. Let $G$ be a nonconstant inner function in $A$. (a) Suppose that $k \in A$ is of the form $k=G \circ h$ on $D$, where $h \in H_{\infty}^{1}$. Then $h$ has an extension to $D \cup \Gamma$ which is continuous. (b) Let $h_{1}, h_{2} \in A_{1}$. Consider the set

$$
S=\left\{z \in D \cup \Gamma \mid h_{1}(z)=h_{2}(z)\right\}
$$

Suppose that $h_{1}(S)$ is infinite. Suppose also that $G \circ h_{1}=G \circ h_{2}$. Then $h_{1}=h_{2}$.

Proof. Since $G$ is an inner function and is a member of $A$, it follows that $G$ is of the form

$$
G(z)=e^{i x} \prod_{n-1}^{N}\left(z-z_{n}\right) /\left(1-\bar{z}_{n} z\right)
$$


where the $z_{n}$ 's are (not necessarily distinct) points of $D$. It follows that, given any point $u_{0} \in \Gamma \cup D$, there exists a disk $D_{0}$ about $u_{0}$ and analytic functions $g_{1}, g_{2}, \cdots, g_{n}$ defined on $D_{0}$, such that if $G(w)=u \in D_{0}$ then $w=g_{j}(u)$ for some $j$. Suppose that $u_{0}=k\left(z_{0}\right)$, where $z_{0} \in \Gamma$. Choose a set $W$ containing $z_{0}$ which is open relative to $\Gamma \cup D$ and satisfies $k(W) \subseteq D_{0}$. On $W \cap D$, we have $k=G \circ h$. It follows that for some $j$, $h(z)=g_{j} \circ k(z)$ for all $z \in W$. Thus, $h$ can be extended continuously to $W \cap \Gamma$. A simple compactness argument now shows that $h$ can be extended continuously to all of $\Gamma$.

Consider the set $Y=\left\{z \in D \cup \Gamma \mid G^{\prime}\left(h_{1}(z)\right) \neq 0\right.$ and $h_{1}(z)=$ $\left.h_{2}(z)\right\}$. We will show that $Y$ is open relative to $D \cup \Gamma$. Since $Y$ is nonempty, it will follow that $h_{1}=h_{2}$. Let $z_{0} \in Y$. Since $G^{\prime}\left(h_{1}\left(z_{0}\right)\right) \neq 0$, there exists an open disk $D_{0}$ about $h_{1}\left(z_{0}\right)$ such that $G$ is one-to-one on $D_{0}$. Choose a set $N$, which is open relative to $D \cup \Gamma$, such that $h_{1}(N) \subseteq D_{0}$ and $h_{2}(N) \subseteq D_{0}$. Then, for $z \in N, G\left(h_{1}(z)\right)=G\left(h_{2}(z)\right)$. It follows that $h_{1}=h_{2}$ on $N$.

Proof of Theorem 3.2: By Theorem 3.1, we may write

$$
U f(z)=\int_{H_{T}} f \circ \phi(z) d \mu(\phi)
$$

for all $f \in A$ and all $z \in D$. For each $z \in D$, let

$$
J_{z}=\left\{\phi \in H_{T} \mid \operatorname{Re} U G(z)<\operatorname{Re} G \circ \phi(z)\right\} .
$$

Suppose that for some $u \in D$, we have $c=\mu\left(J_{u}\right)>0$. Define measures $\mu_{1}$ and $\mu_{2}$ on $H_{T}$ by

$$
\begin{aligned}
& \mu_{1}(K)=c^{-1} \mu\left(K \cap J_{u}\right) \\
& \mu_{2}(K)=(1-c)^{-1} \mu\left(K \cap\left(H_{T} \sim J_{u}\right)\right) .
\end{aligned}
$$

By [7, Prop. 1.1], there are operators $U_{1}$ and $U_{2}$ in $R$ such that

$$
U_{i} f(z)=\int_{H_{T}} f \circ \phi(z) d \mu_{i} \quad i=1,2,
$$

for each $f \in A$ and each $z \in D$. (Note that for $f \in A, U_{\mathrm{i}} f$ is not necessarily in $A$.) It follows that

$$
U f(z)=c U_{1} f(z)+(1-c) U_{2} f(z)
$$

for $f \in A$ and $z \in D$. Since $U G$ is an extreme point of $A_{1}$, it is also extreme point of $H_{\infty}^{1}$. (See [3, p. 139].) Thus, we have $U G=U_{1} G=$ $U_{2} G$ on $D$, but 


$$
\begin{aligned}
\operatorname{Re} U G(u) & =\int_{H_{T}} \operatorname{Re} U G(u) d \mu_{1}(\phi) \\
& <\int_{H_{T}} \operatorname{Re} G \circ \phi(u) d \mu_{1}(\phi)=\operatorname{Re} U_{1} G(u),
\end{aligned}
$$

a contradiction. It follows that for each $z \in D$ we have $\mu\{\phi \mid \operatorname{Re} U G(z)<\operatorname{Re} G \circ \phi(z)\})=0$. Similarly, we can show that

$$
\mu(\{\phi \mid \operatorname{Re} U G(z)>\operatorname{Re} G \circ \phi(z)\})=\mu(\{\phi \mid \operatorname{Im} U G(z) \neq \operatorname{Im} G \circ \phi(z)\})=0 .
$$

Thus, $U G(z)=G \circ \phi(z)$ for all $\phi$ in the support of $\mu$. It follows that the support of $\mu$ consists of finitely many functions $\phi_{1}, \cdots, \phi_{m} \in H_{T}$, where each $\phi_{i}$ satisfies $G \circ \phi_{i}=U G$ on $D$. By Lemma 3.1, each $\phi_{i}$ is continuous on $D \cup \Gamma$. Thus, there exist positive numbers $c_{1}, \cdots, c_{m}$ such that $\Sigma c_{i}=1$ and

$$
U f(z)=c_{i} f \circ \phi_{i}(z)
$$

for each $f \in A$ and each $z \in D \cup \Gamma$. For $z \in \Gamma(T)$, we have $\phi_{i}(z)=$ $\hat{T}(z)$ for $i=1,2, \cdots, m$. It follows by Lemma 3.1, that $\phi_{1}=\phi_{2}=\cdots=$ $\phi_{m}$. Hence $U \in F_{1}(T)$.

REMARK. Theorem 3.2 provides a possible approach to the problem of finding an isometry $T$ such that $T 1=1$ and $E_{1}(T)$ is not the weak operator closure of $\operatorname{cov} F_{1}(T)$. If an isometry $T$ can be found such that: $T 1=1, T$ is not of the form (1), and $T G$ is an extreme point of $A_{1}$ for some nonconstant inner function $G \in A$, then it will follow from Theorem 3.2 that $T \notin$ weak operator closure of $\operatorname{cov} F_{1}(T)$.

\section{REFERENCES}

1. N. Dunford and J. T. Schwartz, Linear Operators, Vol. I, Interscience, New York, 1958.

2. E. Hille, Analytic Function Theory, Vol. I, Blaisdell, New York, 1959.

3. K. Hoffman, Banach Spaces of Analytic Functions, Prentice Hall, Englewood Cliffs, New Jersey, 1962.

4. R. V. Kadison, The trace in finite operator algebras, Proc. Amer. Math. Soc., 12 (1961).

5. J. N. McDonald, Isometries of function algebras, Illinois J. Math., 17, No. 3, pp. 579-583.

6. P. D. Morris and R. R. Phelps, Theorems of Krein-Milman type for certain convex sets of operators, Trans. Amer. Math. Soc., 150 (1970), 183-200.

7. R. R. Phelps, Lectures on Choquet's Theorem, Van Nostrand, 1966.

8. R. Rochberg, Which linear maps of the disk algebra are multiplicative, Pacific J. Math., 38 (1971).

9. W. Rudin, Boundary values of continuous analytic functions, Proc. Amer. Math. Soc., 7 (1956).

Received February 10, 1972 and in revised form February 2, 1974. Part of the work presented here was done while the author was receiving an Arizona State University faculty-grant-in-aide. 


\section{CONTENTS}

Zvi Artstein and John A. Burns, Integration of compact set-valued functions

J. A. Beachy and W. D. Blair, Rings whose faithful left ideals are cofaithful

Mark Benard, Characters and Schur indices of the unitary reflection group $[321]^{3}$

H. L. Bentley and B. J. Taylor, Wallman rings ............................. 15

E. Berman, Matrix rings over polynomial identity rings II ............... 37

Simeon M. Berman, A new characterization of characteristic functions of absolutely continuous distributions ........................ 323

Monte B. Boisen, Jr. and Philip B. Sheldon, Pre-Prüfer rings ............ 331

A. K. Boyle and K. R. Goodearl, Rings over which certain modules are injective

J. L. Brenner, R. M. Crabwell and J. Riddell, Covering theorems for finite nonabelian simple groups. $V$

H. H. Brungs, Three questions on duo rings .................................... 345

Iracema M. Bund, Birnbaum-Orlicz spaces of functions on groups ....351

John D. Elwin and Donald R. Short, Branched immersions between 2-manifolds of higher topological type

J. K. Finch, The single valued extension property on a Banach space ................................................................................. 61

J. R. Fisher, A Goldie theorem for differentiably prime rings ............ 71

Eric M. Friedlander, Extension functions for rank 2, torsion free abelian groups ...................................................................... 371

J. Froemke and R. Quackenbusch, The spectrum of an equational class of groupoids

B. J. Gardner, Radicals of supplementary semilattice sums of

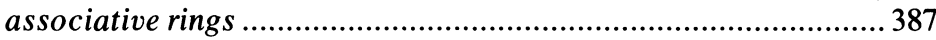

Shmuel Glasner, Relatively invariant measures ...............................393

G. R. Gordh, Jr. and Sibe Mardešić, Characterizing local connectedness in inverse limits...

S. Graf, On the existence of strong liftings in second countable topological spaces

S. Gudder and D. Strawther, Orthogonally additive and orthogonally increasing functions on vector spaces ........................................427

F. Hansen, On one-sided prime ideals .......................................... 79

D. J. Hartfiel and C. J. Maxson, A characterization of the maximal monoids and maximal groups in $\beta x$.

Robert E. Hartwig and S. Brent Morris, The universal flip matrix and the generalized faro-shuffle 


\section{Pacific Journal of Mathematics}

Vol. 58, No. 1

March, 1975

John Allen Beachy and William David Blair, Rings whose faithful left ideals are cofaithful .................................... 1

Herschel Lamar Bentley and Barbara June Taylor, Wallman rings ........ 15

Elizabeth Berman, Matrix rings over polynomial identity rings. II ...... 37

Ann K. Boyle and Kenneth R. Goodearl, Rings over which certain modules are injective ................................. 43

J. L. Brenner, Robert Myrl Cranwell and James Riddell, Covering theorems for finite nonabelian simple groups. $V \ldots \ldots \ldots \ldots \ldots \ldots \ldots \ldots \ldots$

James Kenneth Finch, The single valued extension property on a Banach

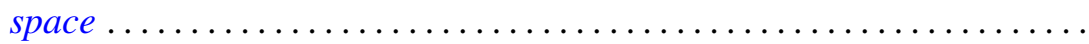

John Robert Fisher, A Goldie theorem for differentiably prime rings........ Friedhelm Hansen, On one-sided prime ideals .................... Jon Craig Helton, Product integrals and the solution of integral equations..........................................

Barry E. Johnson and James Patrick Williams, The range of a normal

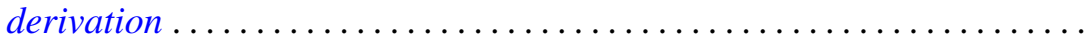

Kurt Kreith, A dynamical criterion for conjugate points ............ 123

Robert Allen McCoy, Baire spaces and hyperspaces .............. 133

John McDonald, Isometries of the disk algebra ................ 143

H. Minc, Doubly stochastic matrices with minimal permanents ......... 155

Shahbaz Noorvash, Covering the vertices of a graph by vertex-disjoint paths. ...

Theodore Windle Palmer, Jordan *-homomorphisms between reduced Banach*-algebras

Donald Steven Passman, On the semisimplicity of group rings of some

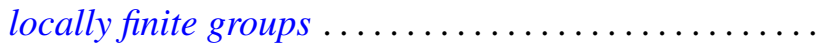

Mario Petrich, Varieties of orthodox bands of groups .

Robert Horace Redfield, The generalized interval topology on distributive

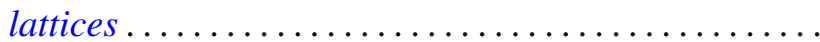

James Wilson Stepp, Algebraic maximal semilattices .... . .

Patrick Noble Stewart, A sheaf theoretic representation of rings with Boolean orthogonalities ........................

Ting-On To and Kai Wing Yip, A generalized Jensen's inequality......... 255

Arnold Lewis Villone, Second order differential operators with self-adjoint

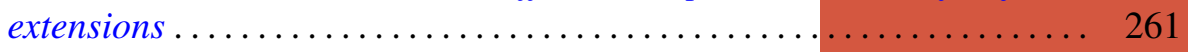

Martin E. Walter, On the structure of the Fourier-Stieltjes algebra ....... 267

John Wermer, Subharmonicity and hulls .................... 283

Edythe Parker Woodruff, A map of $E^{3}$ onto $E^{3}$ taking no disk onto a

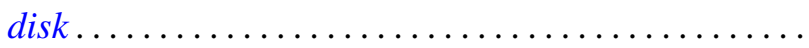

